\title{
Optimizing radiation dose and imaging time with conventional myocardial perfusion SPECT: Technical aspects
}

\author{
Piotr Slomka, PhD, ${ }^{\mathrm{a}, \mathrm{b}}$ and Guido Germano, PhD, MBA ${ }^{\mathrm{a}, \mathrm{b}}$ \\ a Department of Medicine, Cedars-Sinai Medical Center, Los Angeles, CA \\ ${ }^{b}$ David Geffen School of Medicine, UCLA, Los Angeles, CA
}

Received Feb 18, 2016; accepted Feb 18, 2016

doi: $10.1007 / \mathrm{s} 12350-016-0455-\mathrm{x}$

\section{See related article, pp. 876-887}

In a quest to reduce the radiation dose and imaging time for myocardial perfusion imaging (MPI), nuclear cardiology vendors have developed new dedicated cardiac scanners and new software reconstruction methods incorporating resolution recovery attenuation and scatter correction. Although the new dedicated cardiac scanners for nuclear cardiology offer superior imaging characteristics and potential to dramatically reduce time and dose, the relatively high price of the new scanners and the existence of a large installed base of conventional equipment create a barrier to rapid dissemination of this technology. On the other hand, advanced software reconstruction methods, especially those based on resolution recovery only (not requiring attenuation correction maps) could be deployed simply as a software upgrade to most existing SPECT scanners. Thus, it is of great interest to explore how such a software-only approach can help us in optimizing the imaging time or the radiation dose for MPI.

In this issue of Journal of Cardiology, Lecchi et $\mathrm{al}^{1}$ evaluate the performance of one particular implementation of reconstruction with resolution recovery (Astonish $^{\mathrm{TM}}$, Philips), which incorporates the collimator information and proprietary filtering in the iterative reconstruction loop. ${ }^{2}$ Lecchi et al ${ }^{1}$ test low-dose imaging with Astonish by acquiring three simultaneous

Reprint requests: Piotr Slomka, Department of Medicine, Cedars-Sinai Medical Center, 8700 Beverly Boulevard, Los Angeles, CA 90048; slomkap@cshs.org

J Nucl Cardiol 2017;24:888-91.

$1071-3581 / \$ 34.00$

Copyright (c) 2016 American Society of Nuclear Cardiology. projections with different imaging times $(30,15$, and 8 seconds), for each of the projection views of the conventional dual headed camera (Philips Brightview). The full imaging time for this scan was about 16 minutes (for all three dose levels) and dose was 550$740 \mathrm{MBq}$, dependent on patient weight. They subsequently reconstructed the simulated studies with the Astonish method, however, without the use of attenuation correction or scatter correction. They studied 40 normal and 40 overweight patients in this manner. Although their title refers to quarter-time imaging, this is in fact a very realistic simulation of quarter-and halfdose imaging. The quarter-time scan would actually last 4 minutes.

The authors then evaluate the impact of reduced count statistics on the fully quantitative estimation of summed-stress scores (SSS), summed-rest scores (SRS) end-diastolic volume (EDV), end-systolic volume (ESV), and ejection fraction (EF). In this objective quantitative analysis of these perfusion and function parameters, they found no significant differences even with $1 / 4$ dose scans (the scans with 8 -s acquisition time per projection) for normal patients and no differences for $1 / 2$ simulated dose for obese patients. They use objective quantitative analysis and appropriate simulated protocols, allowing for the evaluation of the effect of the progressively reduced dose in the same patient.

Previous visual studies of perfusion analysis have explored the possibility of imaging with reduced imaging time/dose with different implementations of resolution recovery ${ }^{3,4}$ and also half time on similar Astonish system. ${ }^{5}$ So why is this study of particular interest? There are several reasons: First, in previous reports, the assessment of myocardial perfusion was made largely by visual means. Such visual assessment is inherently biased because it is difficult to blind the reader with regard to the type of the reconstruction or duration of the scan. Further, operator variability and 
experience can confound the final results. Lecchi et al use only quantitative parameters, which is a major strength, as it allows an objective evaluation of the results. In addition, they study overweight and normal weight groups separately, which allows them to evaluate the appropriateness of reducing the radiation dose or time separately in patients with different categories of weight.

\section{TIME VS DOSE}

There is often some confusion with regard to the interchangeability of imaging time or injected dose in nuclear cardiology. Counts in MPI can be reduced due to shorter imaging time or due to lower injected dose. Although the effects are likely to be very similar, they are not exactly the same. For example, in the study by Lecchi et al, the entire acquisition still lasts 16 minutes, but shorter projection views are obtained for each projection angle. In fact, an actual quarter-time imaging acquisition would likely be of somewhat better quality than the simulated quarter dose imaging-however, the only way to correctly compare reduced time imaging to standard time imaging on the conventional scanner would be to rotate the camera around the patient several times. In such shorter scans, the effects of patient motion or patterns of extra-cardiac activity could be very different compared to the longer 16-minute acquisition.

For comparison, a previously published study of half-time imaging with the Astonish system used yet another method of simulation, simply removing some projections/views and reconstructing the data with half the views. ${ }^{5}$ This approach actually also simulated better the reduced dose (not reduced time) as the remaining projections are still acquired over the entire time period. In addition, that method introduces an additional change by reducing the number of projections, which will likely have some additional effects on the imaging characteristics. Thus, the simulation of reduced dose by Lecchi et al is more realistic than that previously published for the Astonish system.

\section{OPTIMAL QUANTIFICATION OF LOW-DOSE PERFUSION}

One detrimental effect of lowering the injected dose or reducing the imaging time is the increased noise and consequently variation of normal uptake, which could be mistaken as a false positive defect. Some of the observed bias in the assessment of perfusion defects (i.e., larger defects with lower dose) in the study by Lecchi et $\mathrm{al}^{1}$ is likely caused by this effect. However, this effect could be possibly mitigated to some extent using "countmatched" databases. The normal database used for the quantification of myocardial perfusion could be built from "matched" low-dose images of normal patients. When such "count-matched" databases are used for perfusion quantification, the noise in test patients would be similar and consequently the software would be "more conservative" in estimating perfusion abnormalities, as compared to using a standard database built from studies with full counts.

In fact, our group studied this phenomenon in a simulation of low-dose imaging for fast MPS cameras and found that the use of such count-matched databases did result in lower bias when quantifying perfusion defects with reduced dose. ${ }^{6}$ In Figure 1, we show the averages and standard deviations (for each of the 17 segments defined by American Heart Association) of two normal myocardial perfusion databases created with the same 14 patient studies, but reconstructed separately as full-dose studies and also as low-dose. While average normal counts are very similar, one can observe large differences in inter-subject variation, especially in the apical area.

\section{FUNCTION QUANTIFICATION OF LOW-DOSE SCANS}

Similarly, the authors observe some bias in function analysis, with some decrease in the end-diastolic volume and increase in the systolic volume, which become significant when going to $1 / 4$ dose in overweight patients. This finding is likely related to the way these computations are made by the software program. Due to increased noise, there is less certainty in edge detection and thus it is likely that some additional algorithmic constraints are invoked in order to preserve the overall robustness of the algorithm. In this case also, count calibration based on the injected dose could be perhaps possible for the ejection fraction values in order to deal with this bias, similar to how it was done for EFs computed from 8- and 16-gate studies. ${ }^{7}$ Ultimately, however, the lowest possible dose will be determined by the maximum tolerable variation in perfusion or function variables. Thus, studies should be performed where reproducibility or variation in perfusion and function quantification is measured and compared at different count levels.

\section{HOW DO WE PROCEED?}

Clearly, establishing the optimal lowest possible imaging time or the optimal dose reduction is an important aim, and can result in significant time and cost savings. Is there more potential to reduce the dose even further than done so far on conventional scanners with software-only methods? We think so. It should be 

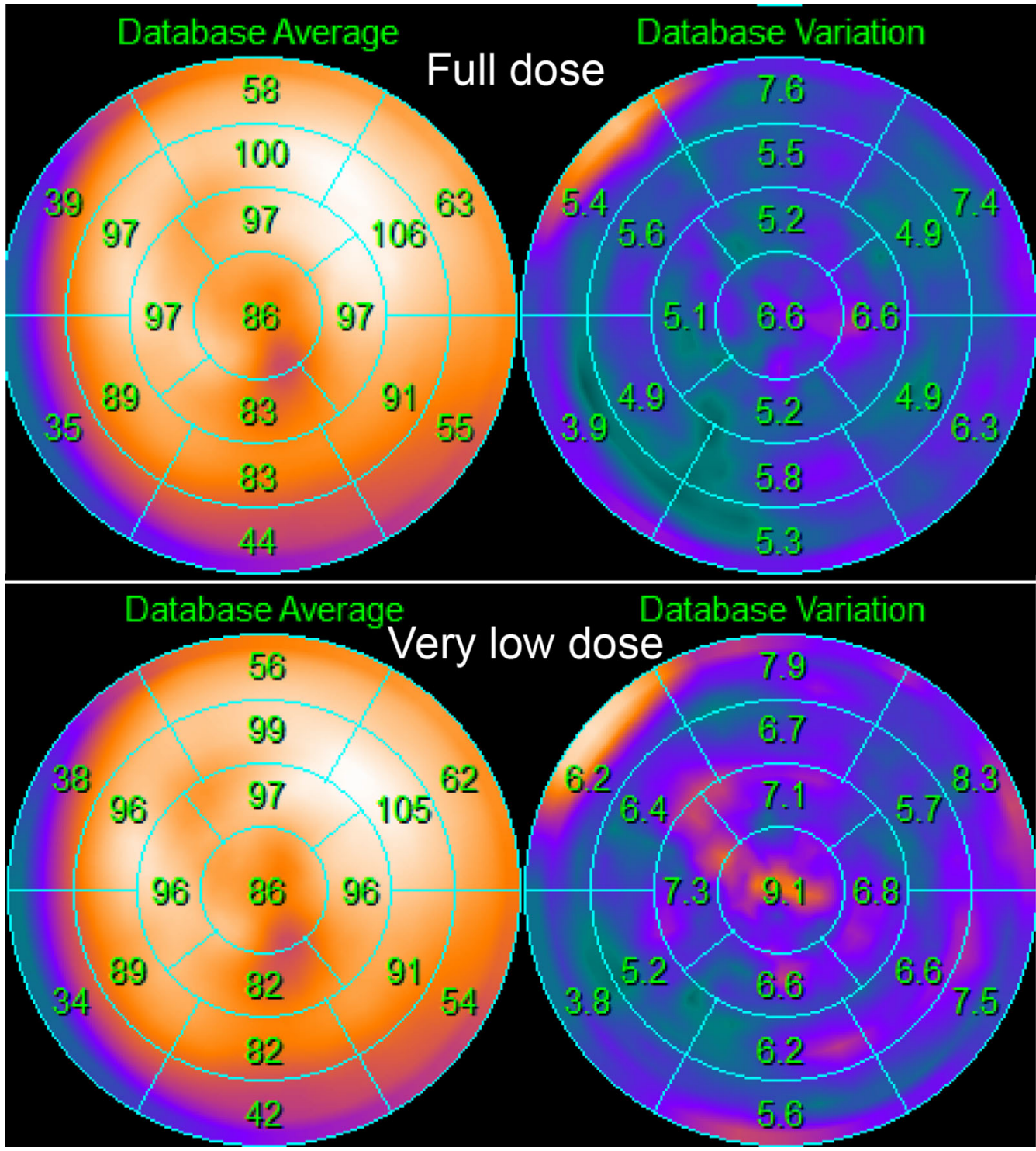

Figure 1. Normal databases (average and variation) for the standard dose (top) and very low dose (125 MBq equivalent) reconstructed from list-mode data of the same 14 patients.

pointed out that the study by Lecchi et al did not use attenuation correction or scatter correction. Adding these components could potentially lead to further imaging improvements. Nevertheless, attenuation correction does require additional hardware and cost, and will add some extra time and potentially additional radiation dose-therefore, the benefits have to be carefully weighted. Further, other advanced methods of reconstruction could be tested. Researchers are developing novel advanced four-dimensional (spatiotemporal) reconstruction models where cardiac motion is taken into account when reconstructing perfusion data. ${ }^{8}$ In a carefully simulated analysis, they have demonstrated that such methods achieve better or comparable performance with $75 \%$ dose reduction than the full-dose data reconstructed with standard reconstruction. Other already available techniques for motion reduction in perfusion scans could also be investigated with regard to the possibility of dose reduction. ${ }^{9}$ Thus, perhaps even lower bounds for the scan time or dose could be established.

While it is clear that there is a need and a high potential to optimize dose and time for MPI on conventional scanners, it is less clear what the most effective approach is to validate it and to introduce it into the clinical practice. We think that with some help from the vendors, the clinical validation and impact can be indeed rapid. For example, it should be possible to 
routinely reduce the count levels post-reconstruction on standard clinical scanners, similar to what can be done with list-mode data for the new fast cardiac SPECT scanners. ${ }^{6,10}$ Right now, such count reductions on conventional scanners have to be simulated by cumbersome modification of routine acquisition methods and require assumption of some predetermined dose reduction "a priori," severely limiting the strategies for clinical validation. If some form of list-mode raw data were routinely made available for the conventional scanners, it would allow rapid clinical validation of the novel reconstruction methods (by just reformatting projections to lower counts), using large numbers of routine clinical studies. Further, automatic perfusion and function analysis could be then optimally "tuned" for the patient-specific counts-again just retrospectively, using the raw data from the routine clinical exams. The optimal lower bounds for time and dose could be then objectively established and validated without significant inconvenience. Such concerted effort on the acquisition, reconstruction, and processing aspects could rapidly enable the nuclear cardiology community to dramatically lower cost and radiation risk, for the large installed base of conventional SPECT scanners.

\section{Disclosure}

Cedars-Sinai Medical Center receives royalties for the quantitative assessment of function and perfusion, a portion of which is distributed to the authors.

\section{References}

1. Lecchi M, Martinelli I, Zoccarato O, Maioli C, Lucignani G, DelSole A. Comparative analysis of full-time, half-time and quartertime myocardial ECG-gated SPECT quantification in normal- weight and overweight patients. J Nucl Cardiol 2015. doi: 10.1007/s12350-015-0382-2.

2. Ye J, Song X, Zhao Z, Da Silva AJ, Wiener JS, Shao L. Iterative SPECT reconstruction using matched filtering for improved image quality. In: 2006 IEEE nuclear science symposium conference record. San Diego: IEEE; 2006. p. 2285-7.

3. DePuey EG, Ata P, Wray R, Friedman M. Very low-activity stress/high-activity rest, single-day myocardial perfusion SPECT with a conventional sodium iodide camera and wide beam reconstruction processing. J Nucl Cardiol 2012;19:931-44.

4. DePuey EG, Bommireddipalli S, Clark J, Leykekhman A, Thompson LB, Friedman M. A comparison of the image quality of full-time myocardial perfusion SPECT vs wide beam reconstruction half-time and half-dose SPECT. J Nucl Cardiol 2011;18:27380 .

5. Venero CV, Heller GV, Bateman TM, McGhie AI, Ahlberg AW, Katten D, et al. A multicenter evaluation of a new post-processing method with depth-dependent collimator resolution applied to fulltime and half-time acquisitions without and with simultaneously acquired attenuation correction. J Nucl Cardiol 2009;16:714-25.

6. Nakazato R, Berman DS, Hayes SW, Fish M, Padgett R, Xu Y, et al. Myocardial perfusion imaging with a solid-state camera: Simulation of a very low dose imaging protocol. J Nucl Med 2013;54:373-9.

7. Germano G, Kiat H, Kavanagh PB, Moriel M, Mazzanti M, Su HT, et al. Automatic quantification of ejection fraction from gated myocardial perfusion SPECT. J Nucl Med 1995;36:2138-47.

8. Jin M, Niu X, Qi W, Yang Y, Dey J, King MA, et al. 4D reconstruction for low-dose cardiac gated SPECT. Med Phys 2013;40: 022501.

9. Slomka PJ, Nishina H, Berman DS, Kang X, Akincioglu C, Friedman JD, et al. "Motion-Frozen" display and quantification of myocardial perfusion. J Nucl Med 2004;45:1128-34.

10. Palyo R, Sinusas A, Liu YH. High-sensitivity and high-resolution SPECT/CT systems provide substantial dose reduction without compromising quantitative precision for assessment of myocardial perfusion or function. J Nucl Med 2016. doi:10.2967/jnumed. 115.164632 . 